\title{
Quantum Liquid Crystal Phases in Fermionic Superfluids with Pairing between Fermion Species of Unequal Densities
}

\author{
Kun Yang \\ National High Magnetic Field Laboratory and Department of Physics, \\ Florida State University, Tallahassee, Florida 32306, USA \\ (Dated: submitted to Phys. Rev. Lett. on July 25, 2005))
}

\begin{abstract}
Superfluidity in fermionic systems originates from pairing of fermions, and Bose condensation of these so-called Cooper pairs. The Cooper pairs are usually made of fermions of different species; thus the most favorable situation for pairing and superfluidity is when the two species of fermions that form pairs have the same density. This paper studies the possible superfluid states when the two pairing species have different densities, and show that the resultant states have remarkable similarities to the phases of liquid crystals. This enables us to provide a unified description of the possible pairing phases, and understand the phase transitions among them.
\end{abstract}

In the Bardeen-Cooper-Schrieffer (BCS) theory for fermionic superfluidity, fermions form Cooper pairs that condense into the zero momentum state. A Cooper pair is often made of fermions of different species; in superconductors they are electrons of opposite spins. Thus the most favorable situation for pairing is when the two species of fermions have the same density, so that there is no unpaired fermion in the ground state. It has been a long-standing fundamental question as to what kind of pairing states fermions can form when the two fermion species have different densities or chemical potentials. An early suggestion was due to Fulde and Ferrell [1], and Larkin and Ovchinnikov [2], who argued that the Cooper pairs may condense into a finite momentum state; this Fulde-Ferrell-Larkin-Ovchinnikov (FFLO) state breaks translation and rotation symmetries. More recently other suggestions have been put forward, including deformed Fermi surface pairing [3, 4, 5] and breached pairing $[\underline{6}$, 7,, 8$]$ states, each with their distinct symmetry properties. Here we show that all these states are different phases of a quantum liquid crystal. This allows us to provide a unified description of all these phases, propose a global phase diagram, and understand the nature of the transitions between neighboring phases.

The issue of pairing between unbalanced fermion species originally arose in superconductors subject to either an external magnetic field or internal exchange field, which gives rise to Zeeman splitting between up- and down-spin electrons that form Cooper pairs. More recently the same issue has been under close scrutiny in the context of pairing and superfluidity in nuclear matter 4], neutron stars [4], and high density quark matter [5, 7, 9], where the unbalance is due to more intrinsic effects like difference in mass of the fermions that form pairs. Perhaps the most promising place where some of these novel phases can be directly observed are trapped cold atom systems 10, 11, 12], where one has the best control of the properties of the constituents and strength of pairing interactions. Thus this is a fundamental issue that is of importance to all branches of physics. To better elucidate the properties of and relations between the various proposed phases, in this work we will use concepts developed in studies of liquid crystals, which is traditionally viewed as a branch of classical physics. The analogy to liquid crystals allows us to determine the global phase diagram of the system, and gain insight into the nature of the transitions between neighboring phases.

We start our discussion with the FFLO state, which has the longest history of studies, and very strong experimental evidence for its existence has been found recently in a heavy fermion superconductor, $\mathrm{CeCoIn}_{5}$ 13, 14]. Following the superconductivity terminology, throughout this paper we will use "spin" indices $\sigma=\uparrow, \downarrow$ to label the two different species of fermions that form Cooper pairs, "Zeeman splitting" $\Delta \mu=\mu_{\uparrow}-\mu_{\downarrow}$ to represent the chemical potential difference between the two species, and "magnetization" $m$ to represent their density difference. When $\Delta \mu \neq 0$, up- and down-spin electrons form Fermi seas with different Fermi momenta $p_{F \uparrow}$ and $p_{F \downarrow}$ in the normal state; it was thus suggested [1, 2] that when pairing interaction is turned on, the electrons with opposite spins on their respective Fermi surfaces would pair up to form a Cooper pair with a net momentum $p \approx p_{F \uparrow}-p_{F \downarrow}$. This results in a pairing order parameter $\Delta(\mathbf{r})$ that is oscillatory in real space, with period $2 \pi \hbar / p$. In general the structure of $\Delta(\mathbf{r})$ is characterized not just by a single momentum $p$, but also by its higher harmonic components. More detailed mean-field study [15] suggested the following real space picture for FFLO state: it is a state with a finite density of uniformly spaced domain walls; across each domain wall the order parameter $\Delta$ (which is real in the mean-field theory) changes sign, and the excess magnetization due to spin unbalance are localized along the domain walls, where $\Delta$ (which is also the gap for unpaired fermions) vanishes; see Fig. 1a. Thus the total magnetization is proportional to the domain wall density. This picture was made more precise by an exact solution in one-dimension (1D) based on bosonized description of spin-gapped Luttinger liquids [16], where the domain walls are solitons of the sine-Gorden model that describes the spin sector; each soliton carries one halfspin. While quantum and thermal fluctuations do not 


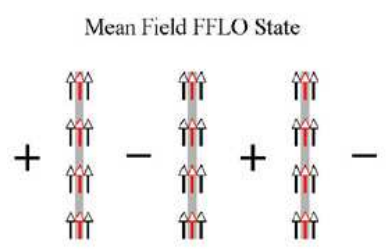

(a)

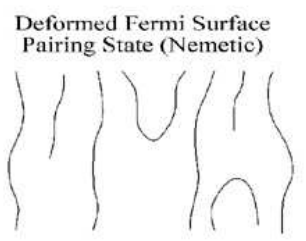

(c)

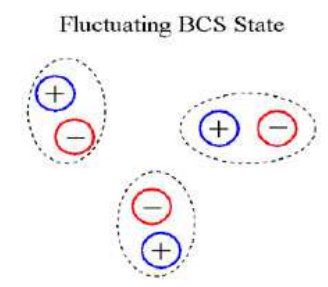

(e)
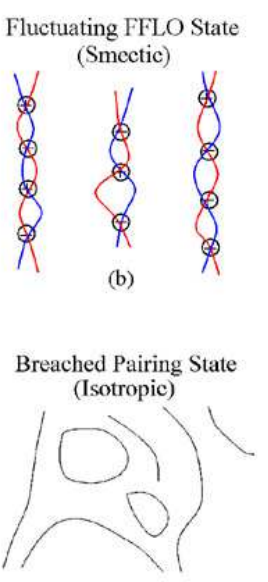

(d)

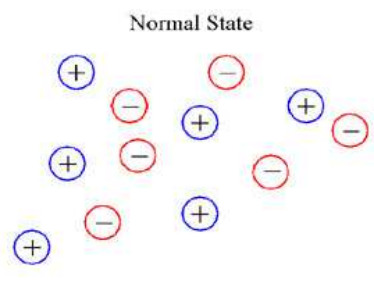

(f)
FIG. 1: (color online). Illustrations of various possible pairing states. a, Domain walls in the mean-field description of the Fulde-Ferrell-Larkin-Ovchinnikov (FFLO) state, where the pairing order parameter (or gap) changes sign and the magnetization is located. $\mathbf{b}$, Domain walls of the FFLO state are replaced by chains of alternating vortices and antivortices once fluctuations are taken into account. The red and blue lines are where the real and imaginary parts of the order parameter vanish respectively, and their intersections are the vortices. The ordered chains break translation and rotation symmetry in a manner identical to the smectic phase of a liquid crystal. c, The nematic phase of the vortex/antivortex chains (or their segments, represented by black lines), which correspond to the deformed Fermi surface pairing (DFSP) state. $\mathbf{d}$, The isotropic phase of the vortex/antivortex chains (or their segments), which correspond to the breached pairing (BP) state. e, The BCS state in which vortices and antivortices (generated by thermal or quantum fluctuations) form closely bound pairs. f, The normal state in which the vortices and antivortices are unbound.

allow true long-range order in $1 \mathrm{D}$, such order can be stabilized by weak interchain couplings 16 . Coming back to isotropic high $\mathrm{D}$ cases, it is clear that the presence and ordering of these domain walls break rotation symmetry, and translation symmetry in the direction perpendicular to the walls, although translation symmetry along the wall remains intact. Thus the symmetry properties of the FFLO state is identical to that of the smectic phase of liquid crystals (smectic-A phase to be more precise) 17].

The domain wall picture described above needs to be modified when fluctuations above mean-field theory are taken into account. In mean-field theory the pairing or- der parameter $\Delta$ is real; it thus vanishes along domain walls which are lines in $2 \mathrm{D}$ and sheets in $3 \mathrm{D}$. In the presence of fluctuations however $\Delta$ is complex, thus it only vanishes where the real and imaginary parts of $\Delta$ are simultaneously zero (see Fig. 1b); these points in 2D and lines in 3D are nothing but the familiar vortices. Thus in the presence of fluctuations the mean-field domain walls are unstable and replaced by chains that are made of alternating vortices and antivortices (see Fig. $1 b$ ), and the FFLO state is a state in which these chains are generated by the Zeeman splitting, and they line-up to break the translation symmetry perpendicular to the chain direction 18.

The appearance of these vortex-antivortex chains when the fermionic superfluids are subject to a sufficiently large Zeeman splitting can also be understood from the following consideration. Zeeman splitting tends to generate spin-polarized quasiparticles; in a uniform superfluid state this can happen only when the splitting is large enough to overcome the quasiparticle gap. On the other hand it is known 19] that the quasiparticle excitations are gapless inside the vortex core; thus the vortices are easily polarizable, making their presence energetically favorable when Zeeman splitting is present. The net vorticity has to remain zero so that equal numbers of vortices and antivortices must be generated, and since vortices repel/attract each other when they have the same/opposite vorticity, they naturally form an alternating pattern. This naturally leads to the vortex chain configurations discussed above.

When quantum and/or thermal fluctuations are sufficiently weak, these infinitely-long vortex/antivortex chains line up and form an ordered smectic phase that breaks translation symmetry along the direction perpendicular to the chains and rotation symmetry, as illustrated in Fig. 1b; this corresponds to the FFLO state in the presence of fluctuations. In classical liquid crystals it is known that as one increases thermal fluctuations, the broken symmetries of the smectic phase are restored in the following sequence 17]: the translation symmetry is restored first when the smectic melts into a nematic that breaks the rotation symmetry only, and then the nematic melts into an isotropic liquid that has no broken symmetry. We thus expect the same sequence of phases and phase transitions occur in superfluids with unbalanced fermion pairing, as we increase the strength of either thermal or quantum fluctuations. More specifically, as fluctuations increase, these infinitely long vortex/antivortex chains can break into segments so that dislocations can appear in the lattice formed by these chains; when such dislocations proliferate and unbind the translation symmetry is restored, although (segments of) the chains are still aligned in a preferred direction, so that the rotation symmetry remains broken (see Fig. 1c for an illustration). This is the analog of the nematic phase, which has the same symmetry as the deformed Fermi sur- 
face pairing (DFSP) state $3,[4,[5]$; we thus identify the DFSP state as the nematic. The chains (or their segments) will lose their orientation alignment upon further increasing fluctuations (see Fig. 1d for an illustration), thus restoring the rotation symmetry (through proliferation of disclinations); this isotropic liquid phase is thus identified with the breached pairing (BP) phase 6,6 , 8] . While all broken spatial symmetries are restored by fluctuations at this point, these phases are still superfluid phases with a spontaneously broken U(1) gauge symmetry, as long as the vortices and antivortices are bound together to form chains or their segments. Further increasing fluctuations will eventually break the segments apart into unbound vortices; this is the normal phase in which the $\mathrm{U}(1)$ gauge symmetry is also restored (see Fig. 1f).

It is clear that the fundamental similarity among the three different superfluid phases discussed above is the appearance of the vortex/antivortex chains (or their segments) in the ground state; they are responsible for accommodating the excess magnetization or unbalance between the fermion species. This is very different from the BCS phase. While in the BCS phase (or ordinary superfluids due to simple Bose condensation as described by the XY model) thermal or quantum fluctuations can still generate vortex excitations, they appear as bound pairs each made of a vortex and an antivortex (see Fig. 1e), instead of forming chains or segments of chains.

Based on the discussion above, we propose a phase diagram in 3D (Fig. 2) for pairing between unbalanced fermion species, assuming the Zeeman splitting $\Delta \mu$ is strong enough to destabilize the BCS state, yet not strong enough to eliminate pairing and superfluidity at $T=0$. For pairing fermions with the same mass, this requires $\Delta \mu \sim \Delta_{0}$, where $\Delta_{0}$ is the pairing gap at $T=0$ and $\Delta \mu=0$; it parametrizes how much energy it costs to break a Cooper pair. We use $\Delta_{0}$ as our unit of energy (although it depends on the pairing strength and other parameters itself). As usual thermal fluctuation is controlled by temperature. Quantum fluctuation is controlled by the strength of pairing interaction; it can be measured by the dimensionless ratio $\Delta_{0} / E_{F}$, where $E_{F}=p_{F}^{2} / 2 M$ is the Fermi energy in the absence of $\Delta \mu$ and pairing interaction, and $M$ is the fermion mass. In trapped cold fermionic atom systems the pairing strength can be controlled by tuning the distance to a Feshbach resonance by a magnetic field, where one can explore a wide parameter range from the weak coupling (or BCS) to strong coupling (which corresponds to the Bose-Einstein condensation or BEC of diatom molecules) regimes of superfluidity [20]. As we increase thermal (increasing $T$ ) and/or quantum (increasing $\left.\Delta_{0} / E_{F}\right)$ fluctuations, the smectic phase (that represents the FFLO state) first melts into a nematic (representing the DFSP state), and then yields to the isotropic (representing the $\mathrm{BP}$ state). The experimental detection of these phases

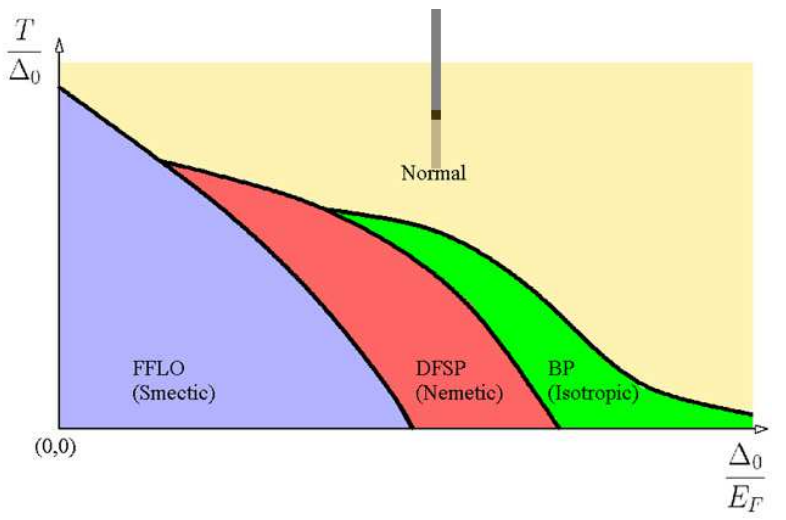

FIG. 2: (color online). Schematic phase diagram for pairing between unbalanced fermion species in 3D. The FFLO state, which is a smectic, is stable at low temperature and weak coupling. As thermal fluctuation (controlled by $T$ ) and quantum fluctuation (controlled by pairing strength and parametrized by the ratio between pairing gap and Fermi energy, $\Delta_{0} / E_{F}$ ) increase, it first melts into a nematic (or the deformed Fermi sea pairing state), which then melts into an isotropic liquid crystal (or breached pairing state). Direct transitions between these superfluid states and the normal state are also possible at high temperature.

should be fairly straightforward in these systems 11, 12]. The nematic-isotropic transition is first order due to a cubic term in the appropriate Landau theory 17$]$. The smectic(-A)-nematic transition is (typically) a continuous transition, although the experimentally observed critical behavior is not yet completely understood 17. Direct transition between these superfluid states and the normal state are also possible. The transition between the $\mathrm{BP}$ and normal phases is in the same universality class as the usual superfluid (or XY) transition, which is usually continuous. On the other hand the FFLO/DFSP to normal transitions should be first order even if the mean-field theory suggests a continuous transition; this is because both spatial and gauge symmetries are broken simultaneously here. Such fluctuation-driven first-order transition has been explicitly demonstrated for the FFLO case recently 21]. The situation is quite different in 2D; there the smectic phase is unstable for any finite temperature, while the nematic-isotropic transition is expected to be of the Kosterlitz-Thouless type 22]. On the other hand the quantum (or $T=0$ ) phase transitions among these phases are poorly understood at this point, and will be the subject of future work.

In the following we present a concrete case of the breached pairing (BP) state, in the strong-coupling (or BEC) regime. In this case unpaired, spin-polarized fermions co-exist with closely-bound fermion pairs (or molecules) that Bose condense; the appropriate fermionic 
many-body wave function takes the form:

$$
|\Psi\rangle=\prod_{|\mathbf{k}| \leq k_{F}^{\prime}} c_{\mathbf{k}, \uparrow}^{\dagger} \prod_{|\mathbf{k}|>k_{F}^{\prime}}\left(u_{\mathbf{k}}+v_{\mathbf{k}} c_{\mathbf{k}, \uparrow}^{\dagger} c_{-\mathbf{k}, \downarrow}^{\dagger}\right)|0\rangle
$$

where $|0\rangle$ is the vacuum state, $k_{F}^{\prime}=\left[6 \pi^{2}\left(n_{\uparrow}-n_{\downarrow}\right)\right]^{1 / 3}$ is the Fermi wave-vector of the excess (or unpaired) fermions, and $u_{\mathbf{k}}$ and $v_{\mathbf{k}}$ (which satisfy $u_{\mathbf{k}}^{2}+v_{\mathbf{k}}^{2}=1$ ) are coherence factors; they are determined by identifying $\Phi_{\mathbf{k}}=v_{\mathbf{k}} / u_{\mathbf{k}}$ as the (momentum space) molecular wave function [23], which in turn can be obtained by solving the two-body Schrodinger's equation with the constraint that $\Phi_{\mathbf{k}}=0$ for $|\mathbf{k}| \leq k_{F}^{\prime}$ :

$$
\frac{\hbar^{2} k^{2}}{M} \Phi_{\mathbf{k}}+\sum_{\mathbf{k}^{\prime}} V_{\mathbf{k k}^{\prime}} \Phi_{\mathbf{k}^{\prime}}=-2|\Delta| \Phi_{\mathbf{k}}
$$

where $V_{\mathbf{k k}^{\prime}}$ is the pairing potential in momentum space. This only leads to a very minor modification of $\Phi_{\mathbf{k}}$ from the genuine two-body problem in the BEC limit, because components with $|\mathbf{k}| \leq k_{F}^{\prime}$ only carry a very small weight for the closely bound state with size much smaller than $1 / k_{F}^{\prime}$; thus the pairing is only "breached slightly" by the presence of the spin-polarized, unpaired fermions. Clearly this is the energetically favored state at strong coupling (compared to the FFLO and DFSP states), as it minimizes the kinetic energy of the unpaired fermions while preserve the pairing correlation for the remaining fermions that do form pairs. The wave function (11) takes a form similar to the one proposed by Liu and Wilczek [6] ; the main difference is that here the unpaired fermions occupy states near the origin in momentum space (instead of near $k_{F}$ of the non-interacting Fermi gas $[\underline{6}]$ ) and form a circular Fermi sea of itself; the origin of the difference is we are considering the strong coupling case while Liu and Wilczek were considering the weak-coupling limit. But they share the same symmetry property (both isotropic), and are therefore different forms of the BP phase.

We close by noting that quantum liquid crystal phases have also been proposed to describe other strongly interacting fermionic systems, including cuprate superconductors 24] and quantum Hall liquids 25, 26].

We thank Nick Bonesteel, Eugene Demler, Akakii Melikidze, Jorge Piekarewicz, and Leo Radzihovsky for useful discussions, and Eddy Yusuf for technical assistance. This work was supported by by National Science Foundation grant No. DMR-0225698.

Note Added - After the present paper has been submitted for publication, the author became aware of three interesting new preprints 27, 28, 29] that address closely related issues from somewhat different angles.
[1] P. Fulde and R. A. Ferrell, Phys. Rev. 135, A550 (1964).

[2] A. I. Larkin and Yu. N. Ovchinnikov, Sov. Phys. JETP 20, 762 (1965).

[3] H. Muther and A. Sedrakian, Phys. Rev. Lett. 88, 252503 (2002).

[4] H. Muther and A. Sedrakian, Phys. Rev. C 67, 015802 (2003).

[5] H. Muther and A. Sedrakian, Phys. Rev. D 67, 085024 (2003).

[6] W. V. Liu and F. Wilczek, Phys. Rev. Lett. 90, 047002 (2003).

[7] E. Gubankova, W. V. Liu, and F. Wilczek, Phys. Rev. Lett. 91, 032001 (2003).

[8] M. N. Forbes, E. Gubankova, W. V. Liu, and F. Wilczek, Phys. Rev. Lett. 94, 017001 (2005).

[9] R. Casalbuoni., G. Nardulli, Rev. Mod. Phys. 76, 263320 (2004).

[10] T. Mizushima, K. Machida and M. Ichioka., Phys. Rev. Lett. 94, 060404 (2005).

[11] A. Sedrakian, J. Mur-Petit, A. Polls, and H. Muther, cond-mat/0504511

[12] K. Yang, cond-mat/0504691

[13] H. A. Radovan et al., Nature 425, 51-54 (2003).

[14] A. Bianchi et al., Phys. Rev. Lett. 91, 187004 (2003).

[15] H. Burkhardt and D. Rainer, Ann. Physik 3, 181 (1994).

[16] K. Yang, Phys. Rev. B 63, 140511 (2001).

[17] P. G. de Gennes and J. Prost, The Physics of Liquid Crystals, 2nd Edition, Oxford University Press, New York (1993).

[18] Under certain circumstances the vortices may also order along the chain direction and break translation symmetry in that direction too; we do not consider the possibility of such (possibly anisotropic) crystal phases here.

[19] C. Caroli, P. G. de Gennes, and J. Matricon, Phys. Lett. 9, 307 (1964).

[20] E. Tiesinga, B. J. Verhaar, and H. T. C. Stoof, Phys. Rev. A 47, 4114 (1993).

[21] D. Dalidovich and K. Yang, Phys. Rev. Lett. 93, 247002 (2004).

[22] J. Toner and D. R. Nelson, Phys. Rev. B 23, 316 (1981).

[23] A. Leggett, in Modern Trends in the Theory of Condensed Matter, edited by A. Pekalski and J. Przystawa (Springer-Verlag, Berlin, 1980), p. 14.

[24] S. A. Kivelson, E. Fradkin, and V. J. Emery, Nature 393, 550 (1998).

[25] E. Fradkin and S. A. Kivelson, Phys. Rev. B 59, 8065 (1999).

[26] Leo Radzihovsky and A. T. Dorsey, Phys. Rev. Lett. 88, 216802 (2002).

[27] C.-H. Pao, Shin-Tza Wu, and S.-K. Yip, cond-mat/0506437

[28] D.T.Son and M.A.Stephanov, cond-mat/0507586

[29] Daniel E. Sheehy and Leo Radzihovsky, cond-mat/0508430 